\title{
Globalización y desigualdades socioterritoriales: la expansión de la periferia metropolitana de Caracas ${ }^{* *}$
}

\begin{abstract}
The analyses of the metropolitan expansion in Latin American cities have put the emphasis on the impacts of the globalization neglecting, in some cases, the decisions of the national economics agents and actors and the trends that were operating before the opening of our economies. The process of globalization of our cities has been partial and rather than produce dual cities has contributed to the conformation of fragmented metropolises. In the Metropolitan Region of Caracas, the sharp socio-territorial heterogeneity due to the restructuring processes is replicated with less intensity in the periphery of the primary city, generating new and diverse socio-territorial inequalities. The economic activities linked to the global economy coexist with the economy of poverty; the livelihood strategies and the ways of life have changed in the context of the mutual interaction among the global and the local, the traditional and the modern.
\end{abstract}

Keywords: metropolitan expansion, socio-territorial heterogeneity, ways of life, Caracas.

\section{Resumen}

Los análisis de la expansión metropolitana en las ciudades latinoamericanas se han ligado en forma directa a los impactos de la globalización, descuidando los efectos de las decisiones de los agentes y actores nacionales y las tendencias que venían operando antes de la apertura de nuestras economías. Los procesos de globalización de nuestras ciudades han sido parciales y han contribuido más a la conformación de metrópolis fragmentadas que de ciudades duales. En la Región Metropolitana de Caracas, la marcada heterogeneidad socioterritorial -producto de los procesos de reestructuración- se reproduce con menor intensidad en la periferia de la ciudad primaria, generando nuevas y diversas desigualdades socioterritoriales. Las actividades económicas ligadas a la economía global conviven con la economía de la pobreza, cambian las estrategias de reproducción y los modos de vida, superponiéndose lo global y lo local en mutua interacción.

Palabras clave: expansión metropolitana, heterogeneidad socioterritorial, modos de vida, Caracas 


\section{Introducción}

I avance de la expansión metropolitana y la conformación de la Región Metropolitana de Caracas (RMC) muestran nuevas caras de las desigualdades socio-territoriales asociadas a la globalización. Mientras en la ciudad primaria se ubican los sectores de mayores ingresos, su periferia "tradicional" se consolida como alternativa residencial para sectores medios vulnerables, y una nueva periferia "emergente" -los Valles del Tuy Medio (VTM)-, además de ser una opción con ventajas para estos últimos, constituye la localización más viable para los sectores medios empobrecidos y para un segmento de los sectores populares.

La saturación de la periferia con mayor desarrollo, conformada por los Altos Mirandinos, Guarenas-Guatire y el Litoral, presiona la expansión metropolitana sobre el Tuy Medio. Mediante el desarrollo de infraestructuras y servicios, proyectos de transporte masivo y de construcción de viviendas privadas y públicas para sectores medios vulnerables y empobrecidos, esta área va adquiriendo un perfil social más heterogéneo respecto al carácter meramente popular y concentrador de pobreza que la caracterizaba en las décadas anteriores. La hipótesis inicial que daba a esa heterogeneidad social una dirección positiva para atenuar las desigualdades socio-territoriales y los fenómenos de sobre-segregación y encierro territorial de los sectores populares va dando paso a una visión más compleja, donde el último de dichos fenómenos, unido a expresiones de aislamiento territorial de los sectores medios, tiende a reproducirse al interior de esta periferia, siguiendo el patrón

Profesores/investigadores del Centro de Estudios del Desarrollo (CENDES) de la Universidad Central de Venezuela. E-mail: cariola@cantv.net, lacabanm@rect.ucv.ve

Una primera versión de este artículo fue presentado como ponencia en el VII Seminario Internacional de la Red Iberoamericana de Investigadores sobre Globalización y Territorio, realizado en Camagüey (Cuba) en noviembre de 2002. de desigualdad y fragmentación del Área Metropolitana de Caracas.

Para avanzar en la comprensión de estos procesos, analizamos las estrategias de reproducción y modos de vida de los sectores populares y sectores medios empobrecidos y vulnerables asentados en esta área, con base en los resultados de investigaciones adelantadas por el Área Urbano Regional de CENDES ${ }^{1}$.

El artículo se compone de tres partes: una primera que ubica a Caracas en el sistema jerárquico de ciudades globales y discute tanto la existencia de una "Caracas ciudad-región global" como de una ciudad dual; la segunda da cuenta de la expansión metropolitana y el papel de los Valles del Tuy Medio en este proceso, y la tercera nos remite a un conjunto de hipótesis sobre la heterogeneidad social, las estrategias y modos de vida en la interfaz periurbana. Finalmente, las reflexiones finales recogen nuestras dudas acerca de la mayor heterogeneidad social y su relación con mejores condiciones de vida en los sectores pobres del área en estudio.

\section{Globalización, ciudad global y ciudad-región}

Las grandes transformaciones económicas y sociales derivadas del proceso de globalización van generando cambios en la configuración y en los modos de vida urbanos, expandiendo nuestras metrópolis hacia la periferia bajo el modelo de ciudad-región compleja y de alto dinamismo, caracterizado por altos niveles de desigualdad socio-territorial. Complejidad derivada tanto de las nuevas funciones y relaciones que la metrópoli asume como de la

Lacabana, M. \& C. Cariola (2001-2002). "Globalización y metropolización: impactos territoriales en la interfaz periurbana de Caracas". Caracas: CENDES-CONICIT. Cariola, C. \& M. Lacabana (20002002). "Transformaciones en el trabajo y reestructuración social. ¿Quiénes son los viejos y los nuevos pobres?". Caracas: CENDES-CDCH/UCV. 
diversidad social y cultural representada por los sectores sociales integrados al sistema mundializado, que comparten desigualmente la ciudad y los beneficios urbanos con aquellos sectores que experimentan diversos niveles de exclusión.

Caracas no escapa a este modelo general, y muestra los rasgos típicos de las metrópolis latinoamericanas de comienzos del siglo XXI. El Área Metropolitana de Caracas (AMC) ha desbordado sus límites urbanos como re- sultado de la progresiva difusión de población, actividades, funciones y relaciones diferenciadas en un territorio mayor, ante la relativa saturación del valle y la constante elevación de los precios de la tierra. Superando barreras topográficas se ha ido conformando la Región Metropolitana de Caracas (RMC), con el valle de Caracas como centro urbano principal que se articula a través de terrenos montañosos con las sub-regiones de su periferia: Altos Mirandinos, Litoral Central, Guarenas-Guatire y los Valles del Tuy Medio (VTM).

Figura 1. Región Metropolitana de Caracas.

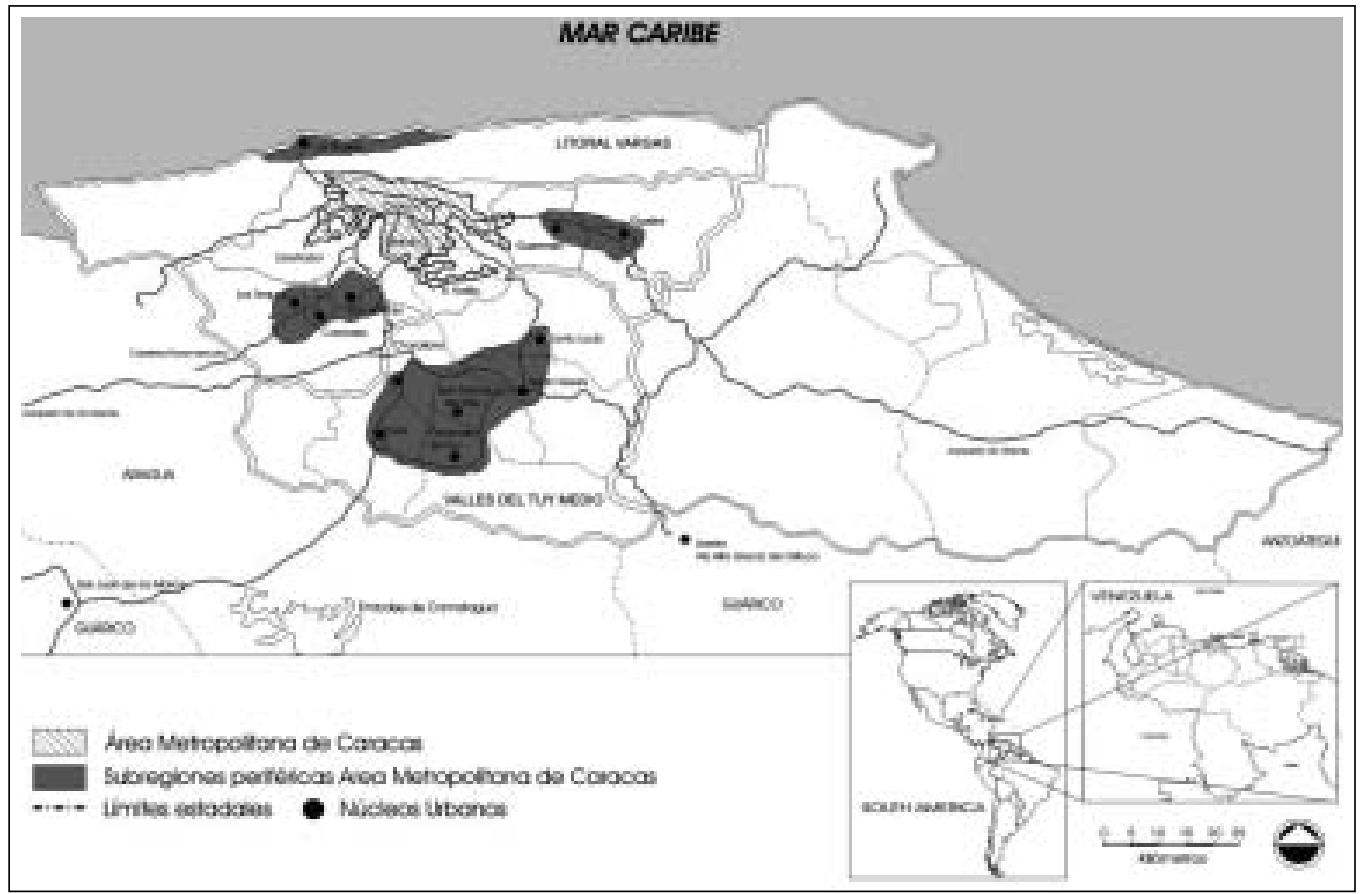

Sin embargo, la RMC no puede asimilarse al concepto de ciudad-región global en el sentido de Scott (2001), dado que una mirada a su estructura productiva nos indica que en la RMC no existen clusters de actividades productivas conectadas a la economía global como las que estos autores refieren, con excepción de algunos servicios avanzados localizados en el distrito de negocios de la ciudad primaria. La RMC tampoco forma parte del mosaico de las ciudades-regiones globales que son núcleos espaciales esenciales de esa economía global, ni es un ac- tor político principal en la escena mundial, aun cuando Caracas tiene un cierto protagonismo por la importancia de Venezuela como país exportador de petróleo. Si bien en la RMC se localizan algunas industrias, otras regiones del país concentran las actividades productivas de bienes, y Caracas tiene una economía fuertemente especializada en servicios. Esto no significa que los problemas referidos a la escala regional metropolitana no están presentes; por el contrario, tal como lo planteamos en este trabajo, son cada vez mayores. 
Ahora bien, si nos atenemos a varios trabajos donde Caracas aparece formando parte de la red de ciudades globales o-dicho de otra forma- inserta en el sistema jerárquico o jerarquía global de ciudades, podemos hablar de Caracas, ciudad en proceso de globalización. Esta adscripción de Caracas al sistema de ciudades globales está relacionada con la localización de servicios avanzados que forman parte de la economía global. De hecho, los trabajos de Friedmann (1986) así como los del Grupo de Loughbourough (1999), donde Caracas aparece en dicha red, se basan en la localización de oficinas de las empresas de cuatro tipos de servicios avanzados (banca y finanzas, contabilidad, publicidad y asesoría legal). En este sentido, las ciudades son el punto de vinculación de las economías nacionales con la economía global a través de su participación en la producción de servicios avanzados (Sassen, 2002).

Esta concentración de servicios avanzados en Caracas tiene estrecha relación con la importancia, tanto nacional como internacional, de la actividad petrolera. Venezuela es el segundo o tercer abastecedor de petróleo de EE.UU., y tiene relaciones privilegiadas con la economía norteamericana ${ }^{2}$. Sin embargo, es a partir de 1989, con las nuevas formas de inserción global de la economía venezolana -que se concretó en la apertura petrolera y minera (con la reincorporación de las empresas petroleras transnacionales al proceso de extracción de crudo), la internacionalización de la banca privada nacional y la privatización de las telecomunicaciones, entre otras actividades económicas- cuando la ciudad se especializa en servicios avanzados y se concentran en ella los headquarters locales de empresas transnacionales.

\footnotetext{
2 Al momento de escribir este artículo, Venezuela se consideraba el tercer abastecedor de petróleo a EE.UU. luego de recuperar la producción después del "paro petrolero" -también conocido como "golpe petrolero"- que sumió al país en una fuerte inestabilidad política y económica, cuyos efectos estarán vigentes por mucho tiempo.
}

El proceso de apertura económica y su impacto sobre la ciudad se da en el marco del cambio en las formas de organización dominante de los flujos transfronterizos y sus actores claves. A partir de los ' 80 prevalecen los procesos de privatización, desregulación y apertura de las economías nacionales a las empresas extranjeras, así como la creciente participación de los actores económicos nacionales en los mercados globales y el papel de las ciudades como territorios estratégicos de las nuevas formas de organización económica y de las actividades estratégicas de la economía global (Sassen, 1999 y 2002).

La concentración en la metrópoli de la población ocupada con un nivel de formación superior, particularmente en los sectores de servicios avanzados, finanzas, comunicaciones, minería y petróleo, demuestra que Caracas ejerce también las funciones técnicas y de control de importantes actividades de producción material, entre las que se destaca la petrolera. Es decir, el desarrollo de los servicios avanzados, telecomunicaciones y finanzas, así como la concentración del control, dirección y gestión de las actividades productivas -principalmente el petróleo-caracterizan el liderazgo económico del AMC como centro nacional y su potencial como ciudad en proceso de globalización. Además, no hay que olvidar que siendo Caracas una ciudad primada, en el sentido de concentrar las élites económicas, políticas y sociales, es también el centro de poder y de decisión, y por lo tanto, localización privilegiada para las empresas.

No son exclusivamente las prácticas de agentes transnacionales, sino en buena medida las prácticas proactivas a la articulación global emprendidas por agentes económicos y actores nacionales, las que explican el proceso de globalización de la ciudad. Este proceso se articula tanto a los cambios económicos asociados a la apertura como también a otros procesos que se venían desarrollando en la metrópoli con la participación de distintos actores, y que han ido creando las bases territoriales para la nueva inserción global. En 
la conformación del nuevo distrito global de negocios de Caracas (Municipio Chacao) fue determinante la participación del capital financiero e inmobiliario nacional, lo que coincide con la afirmación de De Mattos (2002) para las ciudades latinoamericanas: "[...] el capital inmobiliario ha cobrado una mayor importancia en la definición de la orientación y el contenido del desarrollo metropolitano [...]" (8). Una parte importante de las intervenciones urbanas que dieron lugar a un cambio de centralidad de Caracas fueron anteriores al proceso de apertura económica, y por lo tanto, "[...] no debería descartarse la hipótesis de que las tendencias que ahora han cristalizado no pueden considerarse totalmente novedosas, sino como la lógica y previsible culminación de una urbanización capitalista [...]" (De Mattos, 2001: 6).

En el marco del proceso de globalización, la creciente importancia de las metrópolis en los países periféricos ha venido acompañada de la profundización de las desigualdades urbanas. Diversos estudios que ponen el énfasis en los procesos de reestructuración, en la producción y el consumo asociados a la globalización apuntan hacia la conformación de metrópolis duales, donde el avance de procesos de especialización funcional lleva a articular plenamente ciertos fragmentos de la ciudad a la economía global, mientras otros están totalmente marginados de ésta y se vinculan directamente a la economía de la pobreza (Ciccollela \& Mignaqui, 2000; Sassen, 1999). Esta visión se vería reforzada a través del análisis de ciertos efectos socio-territoriales de la descentralización -como componente de la reestructuración política- que dan cuenta de la creciente segmentación institucional y del distanciamiento entre municipios pobres y ricos, que obstaculizan la gobernabilidad de la ciudad y refuerzan la desigualdad socio-territorial.

Sin embargo, el énfasis en los procesos de diferenciación social y socio-territorial como efecto del avance de la exclusión y el empobrecimiento en la ciudad y de la ciudad ponen en entredicho esta visión dual (Cariola \& Lacabana, 2001). En un artículo anterior afirmamos que estamos en presencia de "múltiples ciudades en el territorio metropolitano" (10), lo cual coincide con las precisiones de Marcuse y Van Kempen (2000), quienes proponen la metáfora de la layered city para ejemplificar la superposición de agentes, actores y procesos de dominación y subordinación. Este proceso está claramente descrito en De Mattos (2001), quien también hace referencia al trabajo de Soja sobre la ciudad fractal, fragmentada y polarizada; es decir, configuraciones urbanas que no se corresponden con el modelo de ciudad dual.

Las transformaciones sociales inciden en la forma como se estructura y se vive la metrópoli a través de la dinámica de segregación socio-territorial que ahora cobra una significación cualitativamente diferente, asociada a la yuxtaposición de diversos modos de vida que propician la atomización y el aislamiento residencial, reforzando la fragmentación metropolitana en múltiples territorios desiguales más que en dos escenarios mutuamente excluyentes.

Esta perspectiva enfatiza la dimensión socio-cultural de los procesos de transformación metropolitana. Da cuenta de los cambios en las prácticas socio-culturales y modos de vida de los sectores y grupos sociales residentes en distintos fragmentos de la ciudad a través de sus estrategias residenciales como prácticas adaptativas que conllevan formas particulares de producir y vivir el espacio residencial, incluida la sociabilidad y la construcción de identidad. Cambios en los modos de vida de los diversos sectores sociales en cada fragmento socio-territorial y de la ciudad como cuerpo social frente a la emergencia tanto de valores y pautas de consumo propias de la sociedad globalizada, así como de la violencia y la desestructuración de tejidos socio-culturales asociados al empobrecimiento y desintegración de la sociedad metropolitana (Cariola \& Lacabana, 2001). Es decir, el proceso de globalización de Caracas, pensado como la ar- 
ticulación de procesos globales y locales, no debe entenderse como un impacto sobre un actor, la ciudad, sino como un impacto diferenciado sobre grupos sociales diversos, territorios cambiantes, procesos de dominación y subordinación. Además, en la coyuntura actual de Venezuela, debe entenderse en el marco de los cambios socio-políticos en marcha.

\section{La expansión metropolitana de Caracas y los Valles del Tuy Medio}

Más allá de las consideraciones de las formas de inserción de Caracas en la red mundial de ciudades y en la economía global como un todo, entendemos la RMC como un área funcionalmente integrada, donde existe la ciudad primaria y su interfaz periurbana o subregiones periféricas, hacia donde la ciudad se expande y con la cual interactúa fuertemente en términos de recursos, mercancías, energía y población ${ }^{3}$. La tradicional distinción entre urbano y rural desaparece de hecho y el conjunto pasa a nombrarse en términos generales como urbano, más allá que se distinga claramente la ciudad primaria y su interfaz periurbana. En definitiva, estamos frente a una nueva forma de paisaje urbano (Friedmann, 2001).

En el proceso de expansión metropolitana, mientras Caracas ha ido perdiendo su dinamismo poblacional, las subregiones de la periferia se expanden a un ritmo mayor captando un segmento importante de población excluida del mercado de la vivienda en el centro metropolitano. Las tasas de crecimiento de la población han sido mayores en las sub-regiones metropolitanas que en la ciudad primaria, particularmente en los Valles del Tuy, donde la participación de la población en el conjunto de la RMC se ha ido incrementando en forma sostenida. De hecho, el AMC ha perdido participación relativa en la población nacional y en el conjunto de la RMC, reflejando los procesos de diferenciación territorial.

Cuadro 1. Evolución de la población. Participación relativa 1971-2001.

\begin{tabular}{|c|c|c|c|c|c|}
\hline Años & $\begin{array}{c}\text { Valles del } \\
\text { Tuy/RMC (\%) }\end{array}$ & $\begin{array}{c}\text { Resto } \\
\text { periferia/ } \\
\mathbf{R M C} \\
(\mathbf{\%})\end{array}$ & $\begin{array}{c}\text { AMC/RMC } \\
(\%)\end{array}$ & $\begin{array}{c}\text { AMC/ } \\
\text { Venezuela } \\
(\%)\end{array}$ & $\begin{array}{c}\mathbf{R M C} / \\
\text { Venezuela } \\
\text { (\%) }\end{array}$ \\
\hline $\mathbf{1 9 7 1}$ & 1,1 & 14 & 79,1 & 20,1 & 25,5 \\
\hline $\mathbf{1 9 8 1}$ & 1,9 & 18,0 & 72,4 & 17,8 & 24,6 \\
\hline $\mathbf{1 9 9 0}$ & 3,5 & 20,0 & 64,2 & 14,8 & 23,1 \\
\hline $\mathbf{2 0 0 1}$ & 4,3 & 23,0 & 57,6 & 11,9 & 20,6 \\
\hline
\end{tabular}

Fuente: Elaboración propia con base en INE (Censos Nacionales).

La expansión metropolitana está relacionada principalmente con las lógicas de acumulación del capital inmobiliario y con la dinámica de construcción de viviendas que ha

$3 \quad$ La RMC abarca 17 municipios distribuidos en tres entidades político-territoriales (Distrito Capital, antes Distrito Federal, Estado Miranda y Estado Vargas). Cinco municipios corresponden al Área Metropolitana interna o valle de Caracas, que según la actual constitución conforman un territorio funcional denominado Distrito Metropolitano, y los doce restantes pertenecen a las sub-regiones periféricas. abierto nuevas opciones de asentamiento en la periferia para sectores medios empobrecidos o vulnerables, incapaces de pagar el costo de localizaciones centrales, con las lógicas de localización del capital comercial y de recreación que contribuyen a generar nuevas centralidades suburbanas, y con el desarrollo de sistemas de transporte y de infraestructuras de vialidad que permiten la movilidad de la población en el ámbito interurbano. El centro metropolitano se consolida como mercado de trabajo principal que opera para toda la región 
-e incluso fuera de sus límites-, y da ocupación tanto en las actividades más especializadas y globalizadas como en aquellas actividades informales ligadas a la economía de la pobreza.

La conformación de la RMC asume una diferenciación económico-territorial donde la ciudad primaria se especializa en las actividades económicas más dinámicas y competitivas, como las del terciario superior vinculadas al rol de metrópoli global emergente en el área del Caribe, en tanto la periferia asume de preferencia las funciones ligadas a la producción material, además de la actividad residencial característica de las periferias metropolitanas. Como nítida expresión de la diferenciación socio-territorial, Caracas se reserva para los sectores de mayores ingresos, mientras las sub-regiones periféricas constituyen principalmente el área residencial para los sectores medios empobrecidos y vulnerables (Cariola \& Lacabana, 2001).

También la periferia manifiesta una diferenciación socio-territorial que se ha venido gestando a lo largo de los años. Las tres subregiones de la periferia, con un desarrollo más antiguo y articulado en el ámbito metropolitano debido a diversas condiciones favorables (los Altos Mirandinos, Guarenas-Guatire y el Litoral Central hasta antes del desastre natural), han constituido una alternativa residencial significativa para sectores medios vulnerables e incluso para sectores de ingresos medio altos, y en menor medida, para sectores medios empobrecidos.

Los VTM con condiciones locales de distinto tipo, más adversas y caracterizados por la concentración de grandes urbanizaciones populares producto de anteriores políticas públicas de erradicación, se abrieron como la opción económicamente más favorable para sectores medios empobrecidos desde mediados de los '80. En los últimos años, la saturación de las tres primeras sub-regiones y las restricciones derivadas de los desastres natu- rales de Vargas van dejando a los VTM como el área de expansión más viable de la RMC, no solamente para sectores sociales pobres y empobrecidos, sino también para los sectores medios vulnerables.

\subsection{Los valles del Tuy Medio: de la periferia relegada a la pobreza a la periferia emergente con mayor heterogeneidad social}

El proceso de desarrollo territorial de los Valles del Tuy Medio y su incorporación como área de expansión de la RMC en los últimos 40 años ha pasado por dos grandes etapas.

Una primera y larga etapa desde comienzos de los '60 hasta los primeros años ' 80 da cuenta de su inicio como periferia industrial y residencial de Caracas. Se desarrolló en el contexto del modelo de sustitución de importaciones impulsado por el Estado, de la expansión económica y el proyecto de la Gran Venezuela ligados al "boom petrolero" de los años '70, a un proceso de urbanización muy rápido y a una mejora sostenida de las condiciones de vida de la población, junto con una fuerte movilidad social ascendente (Lacabana \& Cariola, 2001).

En los '60 se comienzan a sentir los efectos de la migración rural urbana y el cambio de uso de las tierras agrícolas en los VTM. La construcción de autopistas que los comunican con Caracas y la electrificación rompen su aislamiento y generan oportunidades para la localización de industrias y el crecimiento de la población en sus principales centros urbanos. Durante los años '70 se implementan políticas de desconcentración espacial del país, que tienen fuerte influencia en los cambios de los VTM y en su relación con Caracas. La prohibición de localizar nuevas industrias en Caracas y la obligación de trasladar las que se consideraban contaminantes, unida a una política de desconcentración industrial que terminó incentivando la localización industrial en un apéndice geográfico del AMC llamado "Area 
B marginal"4, permitió que las empresas se dirigieran fundamentalmente hacia los VTM (Rofman, 1978).

El mecanismo inexorable de expansión de las ciudades se acelera en los años ' 70 como consecuencia de las políticas públicas; se producen cambios en la relación funcional y un nuevo tipo de integración con Caracas que permiten identificar los VTM como parte de la periferia de ésta. Este proceso continúa en una segunda etapa durante los ' 80 y ' 90 , pero con características diferentes y en el marco del agotamiento del modelo de sustitución de importaciones y la apertura progresiva de la economía venezolana al proceso de globalización.

Esta segunda etapa se desarrolla en el contexto de la profunda crisis que afecta a la economía venezolana desde inicios de los ' 80 . En 1989 se implementa un plan de ajuste y apertura de la economía que se planteó como la vía de vinculación con los procesos de globalización y que cambió la composición de la estructura sectorial de la economía, con pérdida de importancia relativa del sector industrial y crecimiento del sector terciario, el cual afectó negativamente la estructura del mercado de trabajo, la distribución del ingreso y dio lugar a un incremento sostenido de la pobreza y la desigualdad. Además, marcó el progresivo abandono del Estado de la estrategia de desarrollo social de largo plazo por las políticas sociales compensatorias y focalizadas (Lacabana \& Cariola, 2001).

Desde mediados de los ' 80 , y hasta avanzados los '90, estas grandes tendencias nacionales se expresan localmente en la concentración de migrantes de Caracas en condiciones de pobreza, con una problemática social ligada a la falta de empleo y el déficit de servicios básicos. La recepción de habitantes de Caracas se da principalmente por la vía de la

4 El Área B Marginal es actualmente parte de la Región Metropolitana de Caracas, y en los '70 recibió incentivos especiales para la localización industrial. construcción de grandes urbanizaciones populares aisladas, que se destinan en un primer momento a damnificados de los barrios del AMC y luego a cubrir necesidades de vivienda de los sectores populares en condición de pobreza, y mediante edificios de viviendas de interés social en los centros urbanos existentes destinadas a trabajadores del sector formal público de Caracas (maestros, policías, empleados y obreros). El desplazamiento de población de bajos recursos generó fuertes conflictos con las autoridades y habitantes locales, por el deterioro en la ya deficiente prestación de servicios públicos y porque se oponían a que los VTM se convirtieran en un área estigmatizada por la concentración de población en condición de pobreza. Sin embargo, la política estatal de vivienda encontró en esta área la posibilidad de desarrollarse por las condiciones topográficas y el bajo costo de los terrenos disponibles y es, en definitiva, la que dio lugar al fenómeno de "sobre-segregación urbana" como expresión de la concentración territorial de la pobreza.

A partir de los ' 90 , el desarrollo territorial del Tuy se da en el marco de la nueva crisis social y política que no sólo replantea y recoge los elementos derivados de los problemas estructurales, sino que incorpora desequilibrios y problemas emergentes. La reestructuración económica y del Estado dejó vigentes transformaciones que tienen relación con una menor intervención estatal en la economía y con la reestructuración del mercado de trabajo que contribuyeron a expandir la pobreza, hacerla más intensa y heterogénea, y a aumentar las desigualdades sociales. Entre los efectos más evidentes de este proceso, además de la profundización y la expansión de la pobreza estructural y la concentración de ingresos en un grupo menor de altos ingresos, se destaca la emergencia de un nuevo segmento social de sectores medios empobrecidos así como el incremento de la vulnerabilidad de sectores medios no pobres (Cariola \& Lacabana, 2001). En la actualidad, más allá de los postulados de la nueva constitución y los esfuerzos del nuevo gobierno por revertir estas tendencias, 
puede decirse que muchas de ellas siguen operando, pero a su vez, nuevos instrumentos de participación y nuevas formas de gestión impulsan el desarrollo local.

Los VTM se han transformado en un foco de atracción de inversiones inmobiliarias para sectores medios en condiciones de vulnerabilidad o ya empobrecidos con medianos y bajos ingresos. Se construyen desde pequeñas urbanizaciones hasta urbanizaciones-ciudades como Valles de Chara, diseñada para albergar a una población de 100.000 personas, con todos los servicios básicos incluyendo educación básica, media y superior, comercio, transporte y recreación. Es decir, esta sub-región de la periferia ha llegado a ser una opción para los sectores medios que no tienen capacidad económica para adquirir vivienda en Caracas y que se apoyan en la Ley de Política Habitacional, la cual promueve viviendas de interés social. Paralelamente, el nuevo gobierno nacional está desarrollando conjuntos habitacionales de mejor calidad, que si bien en parte se destinan a damnificados de la tragedia natural ocurrida en el litoral central de Venezuela en diciembre de 1999, en su mayoría se orientan a un mercado de sectores medios empobrecidos.
Otro elemento central en el desarrollo territorial del Tuy ha sido la construcción del ferrocarril metropolitano a partir de 1995. Este conectará los VTM con Caracas en 14 minutos y su inauguración será en el año 2004. Es un proyecto nacional que responde a los lineamientos del Plan Nacional de Ferrocarriles y al Plan Nacional de Ordenamiento del Territorio y que ha generado fuertes expectativas sobre el crecimiento de la sub-región, tanto entre los empresarios como entre la población local y metropolitana. En este proceso, nuevos actores interesados entran en escena, surgen organizaciones de promoción del desarrollo local que se transforman en actores claves del proceso y al mismo tiempo aumenta el número y la complejidad de los actores primarios y secundarios.

Mientras tanto, la problemática social continúa centrada en el problema del desempleo y en la falta de oportunidades laborales, especialmente para la mujer. Bajas tasas de actividad femenina y altas tasas de desocupación, así como una elevada participación del sector informal en el conjunto de la ocupación contribuyen a la desigualdad socio-territorial respecto a la ciudad primaria.

Cuadro 2. Indicadores del mercado de trabajo 2001.

\begin{tabular}{|l|c|c|c|c|c|c|c|c|}
\hline \multirow{2}{*}{ Áreas } & \multicolumn{3}{|c|}{ Tasa de actividad (\%) } & \multicolumn{3}{c|}{ Desempleo (\%) } & \multicolumn{2}{c|}{ Segmentos (\%) } \\
\cline { 2 - 9 } & Total & Hombres & Mujeres & Total & Hombres & Mujeres & Formal & Informal \\
\hline VTM & 55,6 & 74,4 & 37,2 & 8,6 & 9,9 & 6,2 & 58,7 & 41,3 \\
\hline AMC & 60,8 & 74,3 & 48,4 & 7,0 & 7,9 & 5,9 & 66,6 & 33,4 \\
\hline RMC & 59,9 & 74,5 & 46,2 & 7,3 & 8,2 & 6,0 & 65,8 & 34,2 \\
\hline
\end{tabular}

Fuente: Elaboración propia con base en INE (EHM $2^{\circ}$ semestre 2001).

En los VTM sigue operando el proceso de desindustrialización por el cierre de empresas; el sector de la construcción tiene poca influencia local, pues las empresas traen sus propios trabajadores de fuera; el sector servicios crece, por un lado, en virtud del incremento de las actividades informales, y por otro, se va complejizando progresivamente al ritmo del crecimiento y diferenciación social de la población; los planes oficiales de empleo generan escaso empleo local, caracterizado por la inestabilidad y los bajos ingresos; el desempleo es elevado y Caracas continúa siendo la principal fuente de empleos. 
Puede afirmarse que la confluencia de los impactos de la globalización y las tendencias económicas nacionales con el proceso de metropolización de Caracas, con los grandes proyectos nacionales de infraestructura y con las respuestas locales a los mismos vuelve más heterogéneos económica y socialmente a los VTM, y a su vez, abre la puerta para la posibilidad de implementar una estrategia de desarrollo local que integre la potencialidad de los seis municipios y sus ciudades, dando lugar a nuevas formas de centralidad metropolitana. Sin embargo, en la actualidad esta sub-región periférica se transforma en un escenario de conflictos por el empleo, la tierra y los servicios, por la falta de participación de los gobiernos locales en los proyectos nacionales y a su vez, por la incapacidad de estos gobiernos de responder a las expectativas y demandas de la población.

\section{Expansión periférica con heterogeneidad social: estrategias de sobrevivencia, estrategias de reproducción y modos de vida}

La expansión poblacional de los VTM en los últimos años se ha dado principalmente a través de la migración de sectores medios empobrecidos y vulnerables procedentes de Caracas, que acceden a viviendas construidas por el sector privado a un costo menor que las similares edificadas en otras áreas de la $\mathrm{RMC}$, de la radicación de sectores populares damnificados por el deslave del litoral central en nuevas urbanizaciones del sector público, y de la invasión de tierras por familias de los sectores populares sin vivienda. Nuevos habitantes comparten el territorio con los pobladores locales y con antiguos habitantes de urbanizaciones populares. Con esta incorporación de nuevos habitantes, el territorio periférico se va diferenciando socialmente de manera tal que las localidades más cercanas a Caracas, con mejores servicios, desarrollo de infraestructura y ventajas climáticas se destinan a los grupos más solventes (Cariola \& Lacabana, 2001) 5 .

\subsection{Estrategias residenciales y heterogeneidad social}

Esta nueva característica de mayor heterogeneidad social del Tuy puede verse a través de las estrategias residenciales desplegadas por sectores medios empobrecidos y por sectores populares en la actual coyuntura. Las estrategias residenciales constituyen un componente esencial de las estrategias de reproducción de los sectores medios y de las estrategias de sobrevivencia de los sectores populares que buscan -por la vía de arreglos habitacionales-solucionar necesidades de vivienda que no pueden lograr en el centro metropolitano. El traslado hacia la periferia de Caracas se explica porque en esta área se ofrecen opciones de vivienda privada y pública económicamente viables para estos sectores sociales, mientras la ciudad sigue concentrando las mayores oportunidades de empleo, así como una mayor y más diversa oferta de servicios urbanos.

\subsubsection{Los sectores populares pobres}

En el contexto de reestructuración del mercado de trabajo y de repliegue social del Estado, han prevalecido entre los sectores populares en condición de pobreza estructural estrategias adaptativas de carácter netamente inmediatista, replegadas al ámbito doméstico por la dificultad para construir respuestas colectivas ante el avance de la exclusión y la pobreza. Para los nuevos y viejos habitantes del Tuy pertenecientes a estos sectores sociales, los cambios en las

5 Es el caso de la urbanización-ciudad Valles del Chara, localizada en un área de mayor altura, con mejor clima y más accesible desde la autopista que comunica Caracas con el centro del país, y de las pequeñas urbanizaciones para sectores medios ubicadas en Charallave, el centro urbano más dinámico de los VTM y más cercano a la metrópolis. 
estrategias de sobrevivencia derivados del avance de la crisis social se asocian también a la localización periférica y a los costos que significa vivir alejados del centro metropolitano. Se afectan las prácticas económicas debido a la incidencia del mayor desempleo en el área, los altos costos económicos y de tiempo para trasladarse a desempeñar un empleo en la ciudad que no compensan las remuneraciones obtenidas y la dificultad de incorporar al mercado de trabajo a todos los miembros potencialmente activos de la familia, con la consiguiente contracción del ingreso familiar. Por otra parte, las familias deben recurrir a arreglos domésticos difíciles y conflictivos para cumplir simultáneamente con las diversas obligaciones cotidianas relativas al empleo, cuidado de los hijos, estudio y otras, localizadas en áreas geográficamente distantes.

Expresión del inmediatismo son las estrategias residenciales puestas en práctica por estos sectores sociales para solucionar su problema de vivienda y/o para crear fuentes alternativas de ingresos. En la periferia del Tuy las estrategias residenciales principales contribuyen a la concentración de la población pobre: la invasión de tierras, la consolidación con ampliación de las viviendas en antiguas urbanizaciones populares y el traslado a viviendas de interés social producto de nuevas políticas del Estado en esta periferia.

Un grupo de estos sectores, constituido fundamentalmente por familias allegadas en viviendas de familiares en los VTM o en los densos barrios caraqueños, ha recurrido a la invasión de tierras donde construir su vivienda en forma progresiva. Mientras en Caracas se invaden áreas de alto riesgo aún libres (partes altas de los cerros, terrenos de mucha pendiente, márgenes de quebradas), en los VTM son ocupados generalmente terrenos planos de fácil acceso. También se invaden ciertas áreas de riesgo colindantes con las futuras vías del tren metropolitano, además de terrenos abandonados de viejas urbanizaciones industriales del Estado nunca desarrolladas como tales, áreas que circundan a urbanizaciones populares de vieja data y otros terrenos baldíos municipales o privados. Constituyen ámbitos territoriales de concentración de pobreza extrema, con inexistencia de servicios básicos y graves problemas de desempleo.

Para los sectores populares residentes de las grandes urbanizaciones populares construidas en los ' 80 con el fin de albergar damnificados de los barrios caraqueños, la estrategia residencial ha sido consolidar y ampliar sus viviendas. La subdivisión y/o ampliación de la vivienda es una práctica común de los sectores populares en condición de pobreza que contribuye a la densificación de los asentamientos y al deterioro de la calidad de vida por el incremento del déficit de servicios. Es parte de la estrategia de sobrevivencia de estos sectores dado que la familia extendida, además de dar habitación al familiar que no la tiene, permite optimizar los ingresos o bien incrementarlos mediante el alquiler de partes de la vivienda.

Junto con consolidar la vivienda se ha buscado consolidar las relaciones vecinales, integrándose y construyendo la identidad social en torno al territorio. Las luchas vecinales por lograr mejoras en los servicios y en la calidad de vida, así como el permanente refuerzo de las relaciones familiares entre los grupos domésticos que migraron juntos han contribuido en este proceso, mientras la desestructuración de tejidos vecinales, a causa del avance de la inseguridad y la delincuencia, operaron en sentido contrario.

El aislamiento geográfico y la gran concentración de hogares pobres en estas 
urbanizaciones populares dieron lugar a un fenómeno que denominamos "sobre-segregación urbana”, que produjo cambios en los modos de vida y desajustes importantes no sólo en las estrategias económicas de sobrevivencia, sino en las estrategias cotidianas de los hogares. Particularmente, las escasas oportunidades de empleo han generado el desarrollo de actividades informales ligadas a la economía de la pobreza. Si bien hay una proporción de trabajo formal entre los habitantes locales, el mercado de trabajo se caracteriza por la informalidad y la precariedad de las condiciones de trabajo y los ingresos. Es decir, las oportunidades que se abren para los sectores pobres con estos trabajos dan escasa viabilidad a sus estrategias de sobrevivencia para mejorar su situación de pobreza. Por otra parte, la inseguridad y la violencia cotidiana se incrementaron y las redes vecinales, así como la vida cotidiana, se desestructuraron, generándose conflictos privados (familiares) y comunitarios (públicos). Esta situación, sumada a la falta de oportunidades de trabajo, replegó a estos sectores al ámbito del barrio y la casa, minimizando sus relaciones con los centros urbanos de la periferia y con la metrópolis.

Más allá de la profundización de la pobreza y de la agudización del problema del desempleo local, una parte importante de los grupos familiares ha permanecido en el Tuy y ha superado su problema de desarraigo inicial. También hay indicios de que, a diferencia de los barrios de invasión, estas áreas se han vuelto más heterogéneas. Se transformaron tanto por la llegada de sectores con mayor poder adquisitivo, en general sectores medios empobrecidos que desplazaron a parte de los antiguos habitantes, y por la movilidad social ascendente de un segmento de estos últimos, así como por la invasión de terrenos aledaños impulsada por pobladores $\sin$ vivienda en condición de pobreza extrema ${ }^{6}$. El crecimiento de estas urbanizaciones las convirtió en un mercado para el desarrollo de actividades comerciales y de servicios que han cambiado la imagen urbana, aun cuando persiste la tendencia al encierro territorial y a la concentración de pobreza con falta de oportunidades diversas que conllevan estrategias de sobrevivencia poco viables para la reproducción familiar.

Un grupo importante de sectores populares en el área lo constituyen los damnificados de los desastres naturales del litoral central, que han sido trasladados a grandes urbanizaciones de viviendas de interés social localizadas en terrenos militares incorporados al desarrollo urbano local. La problemática central de estos sectores pasa por el desarraigo territorial que significa el quiebre con todos los lazos previos asociados a su residencia anterior. Ruptura de las relaciones con el empleo, con las redes vecinales, con la vida urbana y los beneficios que la ciudad balnearia tenía para sus habitantes; en fin, ruptura con un modo de vida urbano y una identidad socio-territorial diferentes. Al igual que los habitantes de viejas urbanizaciones populares, las condiciones de pobreza, desempleo y exclusión, unidas a la discriminación que experimentan por parte de la población local, llevan a estos sectores a vivir una especie de encierro dentro de la urbanización.

$6 \quad$ Uno de los casos emblemáticos de la política de erradicación de barrios de Caracas señalada anteriormente es analizado en nuestro estudio sobre la urbanización popular El Cartanal, que se inauguró en 1986 alojando alrededor de 400 familias damnificadas, para ir creciendo hasta estar conformada actualmente por 12 sectores, habitados por aproximadamente 9.000 familias, que suman 50.000 habitantes. Con el transcurso de los años se han desarrollado otras urbanizaciones populares a su alrededor $\mathrm{y}-\mathrm{fun}$ damentalmente- asentamientos informales por invasión de tierras que prácticamente duplican esa población. 
El modo de vida actual evidencia la tendencia de los sectores populares urbanos al encierro territorial dentro de su ámbito residencial y comunitario inmediato, acentuado en la periferia por las condiciones de mayor exclusión del mercado laboral y de los servicios. Es particularmente grave para algunos sujetos sociales. Entre éstos, cabe destacar la extrema exclusión de las mujeres jóvenes, replegadas en su mayoría al ámbito doméstico, dentro de la casa y del barrio (CENDES, 1998). El encierro territorial está asociado a los altos costos del transporte, al avance de la inseguridad y a la transformación de los hábitats populares en espacios económicos. Los altos costos del transporte constituyen una limitación para trasladarse hacia la ciudad, en tanto que la inseguridad con violencia restringe los horarios y las áreas de circulación en una buena parte de los ámbitos residenciales de estos sectores sociales. La falta de oportunidades de trabajo ha llevado, por otra parte, al desarrollo de nuevas formas laborales basadas en la subcontratación, que se apoyan en el trabajo domiciliario y de múltiples actividades informales que tienen a estos ámbitos como mercado 7 .

Como modo de vida, esta particular superposición de espacios residenciales con espacios productivos ligados a la economía de la pobreza contribuye al aislamiento y aleja a los sectores populares de un concepto de vida moderno, evidenciando las desigualdades con otros sectores sociales. "Ese mantenerse circunscrito a un solo tipo de espacio vital urbano, si bien refuerza los modos de vivir la cotidianidad, las redes sociales y las representaciones que se construyen en la vida social,

\footnotetext{
Algunas empresas localizadas en Caracas aprovechan la mano de obra cautiva desempleada en estas urbanizaciones para encargar tareas poco calificadas y sub-pagadas, como el remate de las costuras de ropa, cuyo costo es más elevado si se realiza por trabajadores formales dentro de los establecimientos industriales. Además, la ampliación del mercado local ha permitido que muchas mujeres dejaran empleos de baja calidad en la ciudad y optaran por implementar sus propias actividades informales de venta en pequeña escala en la casa o en la calle.
}

las mantiene restringidas a un ámbito social muy acotado, poco diferenciado y poco diverso, lo cual incide en la pobreza de herramientas para comprender, interpretar y relacionarse con las exigencias del modo de vida urbano moderno contemporáneo [...]" (Guitián, 1998:180).

El encierro territorial responde a la tendencia a concentrar los esfuerzos para la sobrevivencia en el plano doméstico. El deterioro de las condiciones de reproducción material por la caída de ingresos va acompañado, desde el punto de vista subjetivo, con sentimientos de inseguridad y frustración crecientes, dando lugar a respuestas defensivas que se concentran en el ámbito de lo privado. Estas respuestas han sido un problema de los hogares y no de los grupos sociales organizados (Cariola, 2002). Sin embargo, en la coyuntura reciente, esta tendencia al repliegue al ámbito doméstico parece estar cambiando. Surgen nuevas experiencias colectivas y se fortalecen algunas anteriores en el marco de un proyecto que les da coherencia dentro de la propuesta oficial de democracia participativa ${ }^{8}$. Estas experiencias repercuten en una revalorización del ámbito público y pueden incidir positivamente para flexibilizar el mencionado encierro territorial.

\subsubsection{Los sectores medios empobrecidos y vulnerables}

Los sectores medios empobrecidos y vulnerables encuentran en esta periferia la opción de acceder a una vivienda propia que les está negada en la ciudad. Sin embargo, vivir en la periferia tiene diversos costos: desde los económicos (ligados a la pérdida del empleo) hasta los cotidianos (derivados de la dificultad

8 Entre estas experiencias se destacan las de las Mesas Técnicas de Agua, impulsadas por la empresa hidrológica de la Región Metropolitana (Hidrocapital) para asegurar la participación organizada de las comunidades en la solución de problemas de agua, y las de los Consejos Comunales de Planificación, como organizaciones de participación en el desarrollo local. 
de organizar la vida doméstica), pasando por los costos psicosociales (ligados al desarraigo del centro metropolitano).

- Nuevos pobres. Este es un grupo diferenciado internamente, no sólo por los ingresos sino por el capital social y cultural disponible en cada familia, que se traduce en una combinación de prácticas, valores y consumos asociados a distintos sectores sociales. Esta diferenciación es producto de su origen social y de los tránsitos socio-económicos seguidos después del ajuste estructural: unos provienen de los grupos pobres en ascenso del modelo rentista, y la gran mayoría, del descenso de los sectores medios vulnerables. La caída de los ingresos los ha empobrecido recientemente, dado que sus oficios y profesiones se han degradado en términos de ingresos y de prestigio social. Esto los lleva a diversificar sus modos de integración a la sociedad metropolitana buscando en el área pública o semiprivada opciones para la educación y la salud, en la incorporación de la mujer al trabajo formal o informal y en el desarrollo de diversas estrategias residenciales para hacer frente a la exclusión del mercado de la vivienda en la ciudad.

Estas estrategias se mueven desde un extremo similar al de los sectores populares de pobres estructurales, representado por la ampliación y/o subdivisión de la vivienda para alquilar o incorporar la familia extendida, hasta el desplazamiento hacia áreas de menor prestigio dentro de la ciudad, o directamente a la periferia metropolitana. Parte de estos sectores son los que han desplazado a habitantes de las urbanizaciones populares, y otros representan un mercado potencial para viviendas construidas por el Estado en los Valles del Tuy.

El traslado a la periferia está asociado a la búsqueda de seguridad e independencia residencial por parte de familias jóvenes a través de la vivienda propia que no pueden pagar dentro del AMC. En general, conlleva una reorganización de la vida cotidiana con altos costos en términos de tiempos de traslado al empleo en la ciudad, disminución del tiempo libre y de las oportunidades de convivencia familiar, de ruptura de las relaciones vecinales previas y de reorganización de los roles de producción y reproducción al interior de los hogares, ya que muchas de las mujeres deben abandonar transitoriamente sus empleos para dedicarse al cuidado de los hijos. La reorganización de las estrategias económica y cotidiana frecuentemente va asociada al desarraigo respecto del conjunto de relaciones socio-territoriales desarrolladas en la ciudad, y afecta en forma diferente el modo de vida de acuerdo a la homogeneidad o heterogeneidad social del medio residencial. En el primer caso, es más fuerte la tendencia a permanecer en el nuevo ámbito residencial, superando el desarraigo inicial, integrarse a la comunidad socio-territorial y reconstruir la identidad por afinidad con los nuevos vecinos. Al contrario, en comunidades socio-territorialmente heterogéneas con fuerte presencia de sectores populares recientemente ascendidos, junto a segmentos empobrecidos de la clase media, se generan conflictos socio-culturales que conducen al aislamiento, encierro y a la posterior salida del ámbito residencial de los hogares de sectores medios.

Los sectores medios vulnerables. Si bien están en el segmento de no pobres, se consideran vulnerables por sus ingresos, pero tienen un capital social y cultural acumulado que les permite mantenerse fuera de la pobreza, tanto por su nivel económico como por sus hábitos de consumo y su vinculación con el modelo global. En casos extremos se desplazan hacia la periferia metropolitana, donde la oferta privada de vivienda en los VTM apunta a estos segmentos más altos de la empobrecida clase media: profesionales, técnicos y ejecutivos jóvenes. Se construyen urbanizaciones cerradas de edificios bajos sin ascensor, con buena 
calidad de vida derivada de servicios eficientes, seguridad y áreas verdes ${ }^{9}$. La tendencia al autoaislamiento territorial tiene base en la homogeneidad social de estos ámbitos residenciales, cuyos habitantes comparten la condición de ciudadanos con derechos plenos y donde la identidad se construye a partir de la semejanza con el otro, y se minimiza el conflicto asociado a la heterogeneidad sociocultural.

Sin embargo, estos sectores, cuya estrategia residencial viable es la mudanza a la periferia y que acceden a una mejor calidad de vida residencial, la deterioran por el costo económico y por el tiempo de transporte a la ciudad, que se traduce -entre otras cosas-en la reducción de las interacciones con la familia y el entorno. Están incorporados a empleos localizados en Caracas pero también realizan actividades laborales domiciliarias plenamente integradas a la economía global, a través de modernas tecnologías de comunicación que funcionan en forma independiente de la localización territorial. Desde la vivienda, ellos se articulan a redes virtuales, a agentes económicos o a otros sujetos sociales que diversifican su ámbito social, a la vez que se enriquecen los medios para interpretar el modo de vida contemporáneo.

\subsection{Los modos de vida en la periferia metropolitana}

En síntesis, podemos señalar que los modos de vida en la periferia metropolitana se mueven entre diversas tensiones surgidas de la expansión de la RMC hacia áreas aún no bien articuladas funcionalmente como un sistema urbano integrado con identidad propia. Ser habitante del Tuy no es aún ser habitante de Caracas, sino de una periferia específica que le sirve de dormitorio a sectores sociales

9 La gran urbanización Valles del Chara reproduce este concepto integrando en su desarrollo áreas destinadas a instituciones de educación superior, lo que eleva la calidad de los servicios ofrecidos. pobres, empobrecidos y vulnerables, y esta característica marca las tendencias profundas de la vida cotidiana, junto con dar base a nuevas desigualdades socio-territoriales. Identificamos las siguientes tensiones básicas en los modos de vida en la periferia de los VTM.

\subsubsection{Entre el arraigo y el desarraigo socio-territorial}

Vivir en la periferia del Tuy Medio significa vivir la tensión entre el arraigo y el desarraigo territorial. El desarraigo surge al trasladarse desde la ciudad u otra área de la periferia, rompiendo diversas relaciones, modos de vida e identidades asociadas con la localización territorial previa. Este fenómeno del desarraigo afecta a los distintos sectores sociales pero es mucho más fuerte entre aquellos sectores populares trasladados a la periferia en calidad de damnificados, cuya migración ha estado asociada a una situación de emergencia sin opción para elegir, cuya integración se dificulta por la falta de empleo y de servicios básicos e incluso perciben el rechazo de la comunidad local. En el tiempo se observa un progresivo arraigo territorial de la población migrante de estos sectores sociales, que se van identificando como habitantes del Tuy. Para los sectores medios empobrecidos la tensión se expresa entre el desarraigo inicial, asociado más a una pérdida de status social, y a la percepción de transitoriedad ligada a la expectativa de consolidar la residencia futura fuera del área, con los intentos de arraigo para integrarse y construir una identidad relacionada con el territorio. En este sentido operan las nuevas organizaciones de sectores medios que buscan elevar la composición social del área como una vía importante para el desarrollo subregional de los VTM.

\subsubsection{Entre el ser habitante de la periferia o habitante de la ciudad}

Dormir en la periferia y trabajar o estudiar en la ciudad es otra de las tensiones básicas de la vida en los VTM que implica, además de arreglos domésticos costosos y 
conflictivos, dificultades para identificarse como habitante de una ciudad que no termina de integrar funcionalmente esta periferia a su territorio. Los modos de vida de todos los sectores sociales están permeados por la lejanía a los lugares de trabajo, dedicando al transporte recursos y tiempo que deben restar a otras actividades y a compartir la vida cotidiana. El avance de los proyectos de transporte urbano masivo (tren y metro), así como el desarrollo de mejores vías de comunicación con el centro metropolitano, va a incidir positivamente en esta dirección.

\subsubsection{Entre el encierro/aislamiento territorial y la integración a la ciudad}

El modo de vida de los habitantes de la periferia se mueve entre formas de encierro 0 autoaislamiento socio-territorial y la necesaria integración a la ciudad para acceder a sus diferentes beneficios urbanos, incluido el empleo. Para los sectores populares en condiciones de pobreza, el avance de la exclusión, de la inseguridad y de la violencia, unidas a la emergencia de formas de trabajo domiciliario o que tienen al barrio/urbanización como mercado, van profundizando la tendencia al encierro territorial. El modo de vida de los sectores populares se caracteriza por estar circunscrito a su hábitat residencial, generalmente estigmatizado, estableciendo una relación con los centros urbanos más cercanos a través de los servicios locales, y la relación con el centro metropolitano se reduce casi exclusivamente al viaje al lugar de trabajo -cuando se está empleado-, con un disfrute muy limitado de los beneficios que la ciudad ofrece. Los sectores medios empobrecidos y vulnerables tienden a autoaislarse en sus ámbitos territoriales, articulándose débilmente a los servicios locales mientras se integran directamente a los diferentes beneficios disponibles en el centro metropolitano.

\section{Reflexiones finales: ¿hacia donde apunta la mayor heterogeneidad socio- territorial de la periferia del Tuy Medio?}

La RMC se compone de áreas con fuertes procesos de cambio. Los Valles del Tuy Medio cuentan con las mayores posibilidades de expansión en la actualidad; se van articulando más estrechamente a la ciudad primaria y adquiriendo un papel preponderante como área residencial para sectores medios empobrecidos y sectores no pobres vulnerables.

Esta expansión, ligada al desarrollo del ferrocarril metropolitano y a las propias condiciones de los VTM, así como a una amplia oferta inmobiliaria, está dando lugar a una mayor heterogeneidad social y económica. Se desarrollan nuevas actividades económicas formales, especialmente de servicios acordes con los nuevos sectores sociales, pero también actividades informales. Ambas pueden tener un impacto positivo sobre el empleo local, pero insuficiente para absorber la nueva fuerza de trabajo y generar empleos no precarios. La mejora de los sistemas de transporte puede hacer de Caracas un mercado de trabajo viable para muchos de estos sectores y atenuar, de paso, la conflictividad que genera la distancia sobre su vida cotidiana.

La mayor heterogeneidad socio-económica no se ha traducido directamente en una disminución de las desigualdades socio-territoriales. Si bien el fenómeno de sobre-segregación parece haberse superado con la llegada de los nuevos habitantes, con el desarrollo de urbanizaciones para sectores sociales más solventes y con la apertura de nuevas actividades económicas en las viejas urbanizaciones populares, persiste la reproducción de tendencias al encierro territorial de los sectores populares y al autoaislamiento territorial, principalmente por parte de sectores medios vulnerables. La persistencia de estas tendencias se 
enmarca en un patrón de expansión metropolitana marcado por la desigualdad y la fragmentación socio-territorial.

Conflictos de diverso tipo acompañan la expansión metropolitana, y particularmente, los rápidos cambios que se están produciendo en los VTM. Las demandas de viejos y nuevos actores se entrecruzan y potencian en un marco de debilidad de las instituciones locales para dar una respuesta integral a las mismas. Sin embargo, en el contexto de la nueva constitución, una nueva institucionalidad y formas de organización novedosas de los distintos sectores sociales - desde los actores empresariales hasta los sectores populares- se hacen presentes para contribuir con el desarrollo local, la mejora de la calidad de vida y la reducción de las desigualdades.

En este sentido, el desafío es una mayor integración social y urbana de los seis municipios y sus respectivas ciudades con los viejos y nuevos desarrollos residenciales que puede dar lugar a nuevas centralidades metropolitanas, pero además, una mayor integración de la RMC, que contribuya con la reducción de las desigualdades socio-económicas y socioterritoriales.

\section{Referencias bibliográficas}

Cariola, C. (2002). "Pobreza y ciudad: reflexiones desde la investigación". Cuadernos del CENDES, 49.

Cariola, C. \& M. Lacabana (2001). "La metrópoli fragmentada. Caracas entre la pobreza y la globalización". EURE, 27, 80.

CENDES (1998). Diagnóstico cualitativo de la oferta. Motivación al estudio, al trabajo y autoestima de mujeres jóvenes excluidas. Caracas: CENDES, Universidad Central de Venezuela.
Ciccollela, P. \& I. Mignaqui (2000). "Economía global y reestructuración metropolitana. Buenos Aires, ¿ciudad global o ciudad dual del siglo XXI?" Cuadernos del CENDES, 43.

De Mattos, C. A. (2001). "Metropolización y suburbanización" EURE, 27, 80. (2002). "Transformación de las ciudades latinoamericanas. ¿Impactos de la globalización?" EURE, 28, 85.

Friedmann, J. (1986). "The World City Hypothesis". Development and Change, 17. (2001). "Intercity Networks in a Globalizing Era". Scott, A. J. (ed.), Global City-Regions. Trends, Theory, Policy. New York: Oxford University Press.

Guitián, D. (1998). Biografía y sociedad. Una lectura desde la sociología del habitar. Tesis para optar al grado de Doctor en Ciencias Sociales. Caracas: FACES, UCV.

Lacabana, M. I \& C. Cariola (2001). "Household livelihood strategies and social hetegoreneity in the Caracas peri-urban interface". Ponencia presentada en el Seminario Rural-Urban Encounters: Managing the Environment of the Peri-Urban Interface. Development Planning Unit, University College London, Londres, 9-10 noviembre. Marcuse, P. \& R. Van Kempen (eds.) (2000). Globalizing Cities. A New Spatial Order. Oxford: Blackwell Publishers.

Rofman, A. (1978). "Revisión crítica de la política de desconcentración espacial de la economía". Cuadernos del CENDES, 23.

Sassen, S. (1999). La ciudad global. Nueva York, Londres, Tokio. Buenos Aires: Eudeba.

(ed.) (2002). Global network, linked cities. New York: Routledge.

Scott, A. J. (ed.) (2001). Global City-Regions. Trends, Theory, Policy. New York: Oxford University Press. 